Proceedings

\title{
Very Low-Calorie Ketogenic Diet with Vegan Meal Replacements in an Obese Patient with Metabolic Syndrome Modifies Visceral Adipose Tissue Distribution and Taxonomic Composition of Gut Microbiota $^{\dagger}$
}

\author{
Elisabetta Camajani ${ }^{1, *}$, Agnese Persichetti ${ }^{2}$, Savina Contini ${ }^{1}$, Mikiko Watanabe ${ }^{1}$, Renata Risi ${ }^{1}$, \\ Carla Lubrano ${ }^{1}$, Stefania Mariani ${ }^{1}$, Massimiliano Caprio ${ }^{3,4}$, Lucio Gnessi ${ }^{1}$ and \\ Sabrina Basciani ${ }^{1, *}$ \\ 1 Department of Experimental Medicine, University of Rome “La Sapienza”, Rome, Italy \\ 2 Service of Pharmacovigilance, IRCCS-Regina Elena National Cancer Institute, Rome, Italy \\ 3 Department of Human Sciences and Promotion of the Quality of Life, San Raffaele Roma Open University, \\ Rome, Italy \\ 4 Laboratory of Cardiovascular Endocrinology, IRCCS San Raffaele Pisana, Rome, Italy \\ * Correspondence: elisabetta.camajani@uniroma1.it (E.C.); sabrinabasciani@yahoo.it (S.B.) \\ + Presented at the 1st International Electronic Conference on Nutrients - Nutritional and Microbiota Effects \\ on Chronic Disease, 2-15 November 2020; Available online: https://iecn2020.sciforum.net/.
}

Published: 30 October 2020

\begin{abstract}
Short-term interventions based on very low-calorie ketogenic diets (VLCKDs) and meal replacements are a viable option for selected metabolic syndrome patients and/or to those with an altered microbiota composition. In this view, protein source choice is still debated. In fact, scarce and inconsistent data, are available on protein intake from various sources on body weight, composition of gut microbiota and metabolic outcomes in these patients. A 52-year-old man affected by first degree obesity, hypertension, hyperuricemia, hyperinsulinism, hypercholesterolemia, hypertriglyceridemia and altered gut microbiota with an increased Firmicutes/Bacteroidetes ratio, was admitted to our facility. Therefore, a nutritional treatment with VLCKD ( $\leq 800 \mathrm{kcal} /$ day) was set for a 45 days period based on meal replacements containing vegetal proteins. Anthropometric indexes, blood and urine chemistry, body composition, muscle strength, taxonomic composition of the gut microbiome, were assessed at the beginning and after 45 days. A significant reduction of body weight (93 vs. $109 \mathrm{Kg}$ ), BMI (27 vs. $33 \mathrm{Kg} / \mathrm{m}^{2}$ ), blood pressure (120/70 vs. 150/100 vs. mmHg), waist circumference (102 vs. $114 \mathrm{~cm})$, HOMA index (2,3 vs. 9,1), triglycerides (129 vs. $612 \mathrm{mg} / \mathrm{dL})$, total cholesterol (151 vs. $318 \mathrm{mg} / \mathrm{dL}$ ) and HDL cholesterol (48 vs. $32 \mathrm{mg} / \mathrm{dl}$ ) and visceral adipose tissue (599 vs. $793 \mathrm{~g}$ ) was observed at 0-45 days respectively. Importantly, a decrease in the relative abundance of Firmicutes, an increase in Bacteroidetes and Verrucomicrobiae abundance was also observed. These preliminary results suggest that the nutritional treatment with a vegan VLCKD led to a significant weight loss, a marked improvement of metabolic parameters and a restored eubiosis in gut microbiota. VLCKD with vegetal protein have promise as a valuable treatment for obesity, leading to an improvement of metabolic health, also related to a favourble rebalancing of gut microbiota.
\end{abstract}

Keywords: VLCKD; Metabolic Sindrome; gut microbiota

\section{Introduction}


Metabolic Syndrome (MetS) was described for the first time by Gerald Reaven in 1988 [1] and was defined as the association between insulin resistance (IR), hypertension, dyslipidemia, Nonalcoholic fatty liver disease (NAFLD), impaired glucose tolerance and other metabolic abnormalities related to increased cardiovascular risk [2].

Moreover MetS is linked to increase mortality for coronary heart disease (CHD), diabetes and cancer $[3,4]$.

Although the pathophysiological mechanism is still under study, a key factor in the pathogenesis of MetS is represented by IR, a phenomenon prevalent in the obese population and characterized by the resistance of peripheral tissues to insulin action. According to the National Cholesterol Education Program's Adult Treatment Panel III report (NCEP ATP III) it is possible to diagnose metabolic syndrome when at least 3 of the following: Waist circumferences $(W C) \geq 102 \mathrm{~cm}$ man and $\geq 88$ woman; Blood pressure $(\mathrm{BP}) \geq 130 / 85 \mathrm{mmHg}$, or antihypertensive treatment; Triglycerides $(\mathrm{Tg}) \geq 150 \mathrm{mg} / \mathrm{dL}$ or lipid lowering treatment; HDL $<39 \mathrm{mg} / \mathrm{dL}$ man $<50 \mathrm{mg} / \mathrm{dL}$ woman; Glucose $\geq 101 \mathrm{mg} / \mathrm{dL}$ or hypoglycemic treatment [5].

Recent scientific evidence affirms that Very Low Calorie Ketogenic Diet (VLCKD) can be a valid treatment for patients with hyperinsulinism and visceral obesity [6-8].

It has been reported that the factors contributing to MetS are the result of extrinsic factors such as diet and lifestyle and complex host intrinsic factors such as genetics and gut microbiota [9]. In particular it is known that in obese subjects there is a dysbiosis of gut microbiota [10,11]: obesity is characterized by an altered intestinal Bacteroides/Firmicutes ratio, with greater relative abundance of Firmicutes [12]. This modification in gut microbiota composition is associated with greater energy breakdown and absorption from food, higher efficiency in storing fat in adipose tissue, as well as stimulation of metabolic endotoxemia and low-grade inflammation $[9,13]$.

We hypothesized that VLCKD and subsequent modification of gut microbiota is strictly linked to the improvement of MetS.

\section{Clinical Case}

A 52-year-old man affected by first degree obesity, hypertension, hyperuricemia, hyperinsulinism, hypercholesterolemia, hypertriglyceridemia and altered gut microbiota with an increased Bacteroides/Firmicutes ratio, was admitted to our facility. Baseline medication therapy was carvedilol $6.25 \mathrm{mg}$, irbesartan $300 \mathrm{mg}$, hydrochlorothiazide $12.5 \mathrm{mg}$ and allopurinol $300 \mathrm{mg}$.

This patient was screened from March 2019 to June 2019 and was prescribed VLCKD with meal replacements based on vegetal protein $[780 \mathrm{kcal} / \mathrm{day}$, with the following composition in macronutrients, percentage of caloric intake and $\mathrm{g} / \mathrm{kg}$ of ideal BW of proteins (derived by the BMI set at $\left.25 \mathrm{~kg} / \mathrm{m}^{2}\right)$ : carbohydrates $26 \mathrm{~g}(13.5 \%)$, olive oil $20 \mathrm{~g}$ plus $15 \mathrm{~g}$ of lipids from other sources $(40.4 \%)$, proteins $90 \mathrm{~g}(46.1 \%, 1.4 \mathrm{~g} / \mathrm{Kg})$ ] for 45 days. The amount of proteins was within the proposed essential composition of total diet replacements for weight control adjusted for the patients with obesity.

He ate five meals/day [timing was at main meals ( 8 a.m., 1.00 p.m. and 8.00 p.m.), mid-morning and mid-afternoon] containing vegetable protein derived from soya, green peas or cereals and one serving of vegetables with low glycemic index at lunch and dinner. Supplements of vitamins, minerals and omega-3 fatty acids, were provided in accordance to international recommendations (EFSA 2017).

Blood count, electrolytes [chloride, potassium and sodium (indirect ion-selective electrode potentiometry), calcium and magnesium (colorimetric assay)], glucose (enzymatic colorimetric assay), insulin (electrochemiluminescence immunoassay), lipids [(triglycerides, total, HDL and LDL cholesterol) (enzymatic colorimetric assay)], total proteins and albumin (capillary system), C-reactive protein (CRP) (immunoturbidimetric assay) and erythrocyte sedimentation rate (ESR) (capillary photometric assay), plasma creatinine (kinetic colorimetric compensated Jaffe method), blood urea nitrogen (BUN) and uric acid, alanine transferase (ALT) and aspartate transaminase (AST) (enzymatic colorimetric assay) and estimated Glomerular Filtration Rate (eGFR) were determined at baseline and T45. It was also required Oral Glucose Tolerance Test (OGTT). Body weight (BW), height, systolic and diastolic blood pressure (BP), waist circumference (WC), thigh circumference 
(TC) and hip circumference (HC) were measured at T0, and every two weeks. Furthermore, SIRT1 and Insulin Growth Factor-1 (IGF-1) were evaluated through with human SIRT1 ELISA kit (MyBioSource, Cod. GDMBS705558) and IGF1 ELISA kit (human igf1 duoSet elisa R\&D) respectively.

Handgrip strength (HG) was measured through a digital dynamometer (DynEx, Akern, Pontassieve, FI, Italy) at T0 and T45. Three measurements were repeated with dominant and nondominant arms. The highest value measured was recorded. Body composition, total and regional body fat mass (FM), visceral adipose tissue (VAT), fat-free mass (FFM) and lean mass were measured through DEXA (Hologic 4500, Bedford, MA) at baseline and at the end.

Fecal sampling was done using sterile swab (FLmedical, Italy) and tubes (Starlab Group, Italy) in the morning of the day of starting VLCKD and at the end.

Following nutritional therapy, a significant reduction of body weight (93,4 vs. $109 \mathrm{Kg}$ ), BMI (27 vs. $\left.33 \mathrm{Kg} / \mathrm{m}^{2}\right)$, waist circumference $(102$ vs. $114 \mathrm{~cm})$ and blood pressure $(120 / 70$ vs. 150/100) was observed after 45 days (Table 1); the improvement of metabolic parameters led to a reduction of hypertension therapy (reduction of carvedilol and interruption of hydrochlorothiazide). Despite the change in therapy, blood pressure was normal and stable over time. Also, a reduction of HOMA index $(2,3$ vs. 9,1), total cholesterol (151 vs. $318 \mathrm{mg} / \mathrm{dL})$, triglycerides $(129 \mathrm{vs} .612 \mathrm{mg} / \mathrm{dL})$ was observed at 0-45 days respectively (Table 2). At baseline the value of $612 \mathrm{mg} / \mathrm{dl}$ was confirmed and causes of pancreatitis and related diseases were excluded. Was also observed an increase of HDL cholesterol (48 vs. $32 \mathrm{mg} / \mathrm{dL}$ ). DEXA analysis showed a decrease of VAT (599 vs. $793 \mathrm{~g}$ ) (Figure 1). Furthermore, a reduction in IGF-1 circulating levels (100 vs. $140 \mathrm{ng} / \mathrm{mL}$ ) and an increase in SIRT (264 vs. $144 \mathrm{ng} / \mathrm{mL}$ ) were observed after 45 days.

Table 1. Participant characteristics of anthropometric measurements at baseline (T0) and every two weeks of VLCKDs.

\begin{tabular}{lcccc}
\hline & T 0 & T 15 & T 30 & T 45 \\
\hline Height (cm) & 181 & & & \\
Weight (Kg) & 109 & 101.7 & 97.1 & 93.4 \\
Body Mass Index $\left(\mathbf{K g} / \mathbf{m}^{2}\right)$ & 33 & 31 & 29.6 & 28.4 \\
Waist Circumference (cm) & 114 & 108.5 & 105.8 & 101 \\
Hips Circumference (cm) & 115 & 113.5 & 112.8 & 109.7 \\
Systolic Body Pressure (mmHg) & 150 & 120 & 120 & 120 \\
Diastolic Body Pressure (mmHg) & 100 & 90 & 80 & 70 \\
Heart Rate & 60 & 60 & 64 & 70 \\
\hline
\end{tabular}

Table 2. Characteristics of blood test and body composition parameters at baseline (T0) and after 45 days (T 45) of VLCKDs.

\begin{tabular}{lcc}
\hline & T 0 & T 45 \\
\hline Fasting Glycemia (mg/dL) & 114 & 92 \\
Fasting Insulin $(\mu \mathrm{IU} / \mathbf{m L})$ & 32.2 & 10.1 \\
Glycemia 120' $(\mathbf{m g} / \mathbf{d L})$ & 126 & 101 \\
Insulin 120' $(\mu \mathrm{IU} / \mathbf{m L})$ & 506 & 45.1 \\
HOMA IR & 9.1 & 2.3 \\
Hb1AC (\%) & 5.6 & 5.2 \\
BUN (mg/dL) & 40.8 & 33 \\
Creatinine (mg/dL) & 0.8 & 0.8 \\
eGFR & 166.5 & 154.3 \\
Na (mmol/L) & 140 & 138 \\
K (mmol/L) & 4.1 & 4.2 \\
Cl (mmol/L) & 98 & 97 \\
Ca (mg/dL) & 9.7 & 9.7 \\
Mg (mg/dL) & 1.6 & 2 \\
P (mg/dL) & 3.4 & 3.1 \\
\hline
\end{tabular}




\begin{tabular}{lcc}
\hline AST (U/L) & 61 & 41 \\
ALT (U/L) & 92 & 57 \\
Total Cholesterol (mg/dL) & 318 & 151 \\
LDL Cholesterol (mg/dL) & 163 & 77 \\
HDL Cholesterol (mg/dL) & 32 & 48 \\
Triglycerides (mg/dL) & 612 & 129 \\
Uric acid (mg/dL) & 5.7 & 7.7 \\
CRP (mcg/L) & 1900 & 900 \\
ESR (mm/h) & 34 & 33 \\
IGF-1 (ng/mL) & 140 & 100 \\
SIRT 1 (ng/mL) & 144 & 264 \\
HG (Kg) & 35.3 & 36.4 \\
VAT Mass (g) & 793 & 599 \\
\hline
\end{tabular}

Abbreviations: HOMA-IR, homeostasis model assessment- insulin resistance; BUN, blood urea nitrogen; eGFR, estimated Glomerular Filtration Rate; AST, aspartate transaminase; ALT, alanine transferase; CRP, C reactive protein; ESR, erythrocite sedimentation rate; HG, handgrip strength; VAT, visceral adipose tissue.
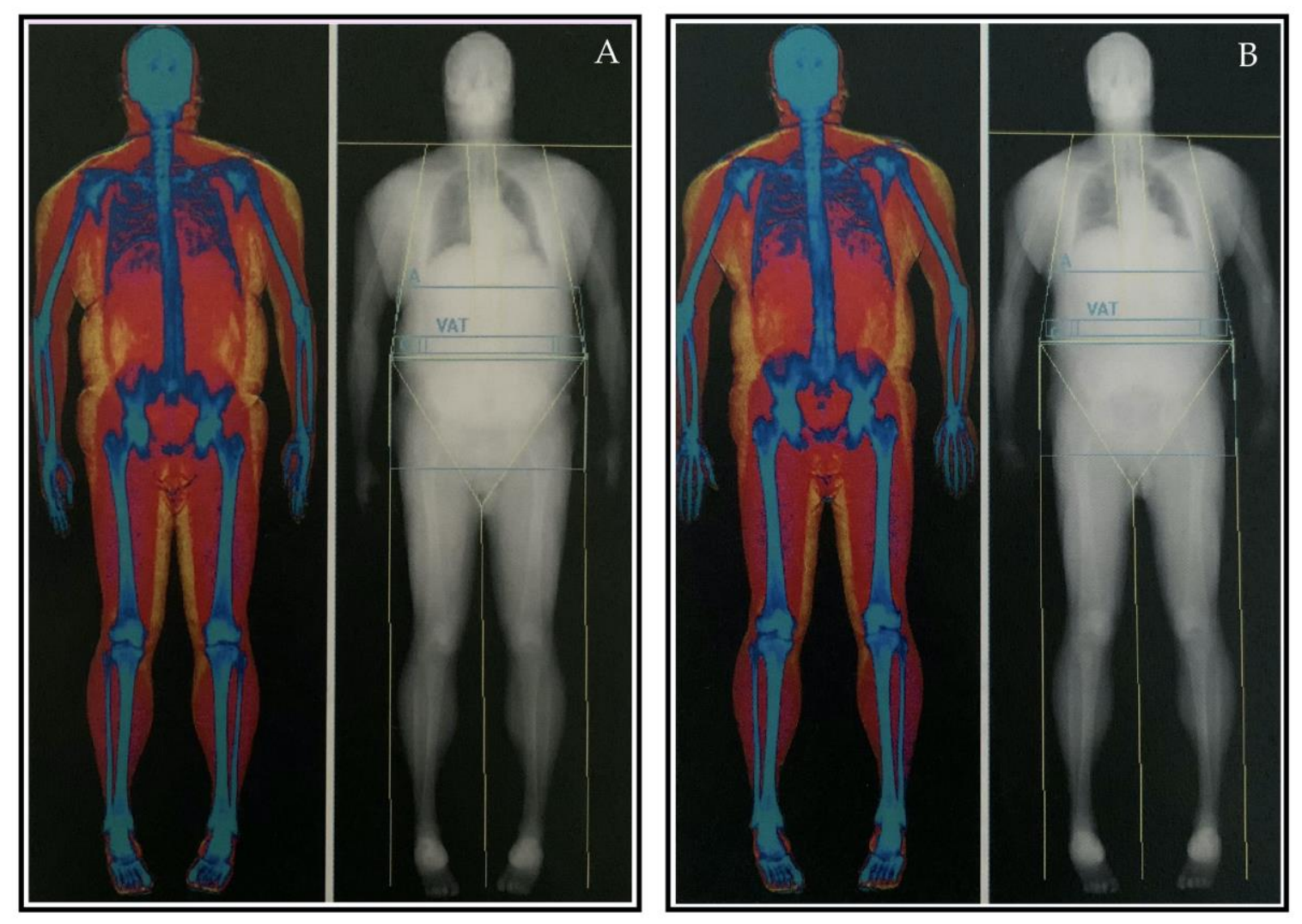

Figure 1. Measurement of VAT by DXA at baseline (Panel A) and after 45 days (Panel B).

Dominant phyla in fecal samples from the patient at T0 were Firmicutes, Bacteroidetes, Proteobacteria, Verrucomicrobia, Fusobacteria and Actinobacteria. Relative abundance of Firmicutes was significantly reduced and Bacteroidetes proportionally increased 45 days after the beginning of VLCKD. The mean relative abundance of Proteobacteria also increased while Actinobacteria decreased (not shown). An increase of Verrucomicrobia was also observed (Figure 2). 

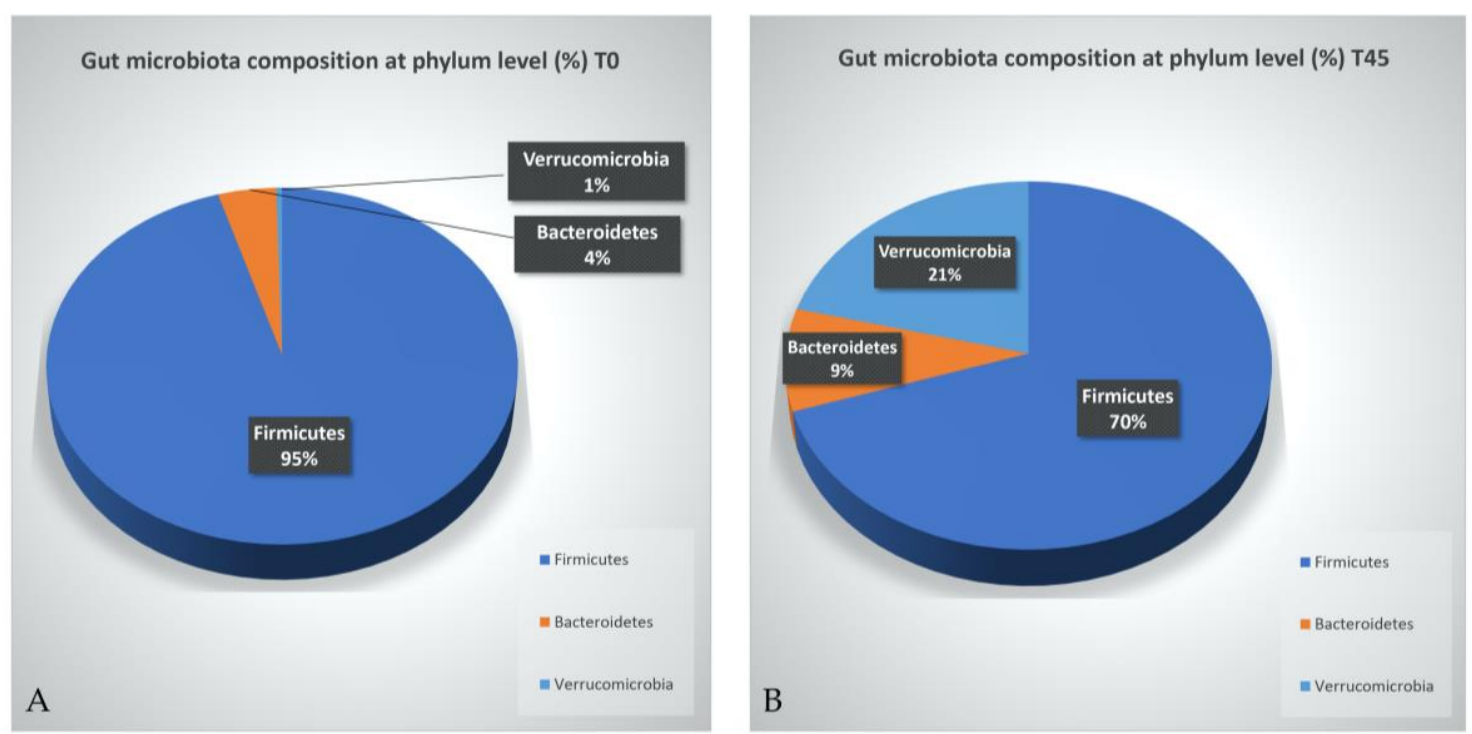

Figure 2. Effect of VLCKD dietary interventions with vegetable protein on the relative abundance of Firmicutes, Bacteroidetes and Verrucomicrobia at baseline (A) and after 45 days (B).

\section{Discussion}

In recent years, an increasing number of studies have focused on VLCKD as a viable dietary approach for obesity, visceral obesity, NAFLD and type 2 diabetes mellitus (T2DM) [14-17].

This case report shows that VLCKD caused a marked reduction of BW (in the range of 15\%), leading the patients from grade I obesity to overweight. It also improves parameters of metabolic syndrome with a subsequent reduction of cardiovascular risk.

An important concern with low carbohydrate diets relies on the potential negative impact on lipid metabolism due to the increased proportion of calories resulting from fat. Clearly, this does not apply to a 45 days VLCKD, for which the daily lipids intake is low as can be inferred by the profound reduction of the circulating cholesterol and triglycerides observed in our patient. Many other mechanisms may contribute to the reduction of circulating lipids; the improvement of insulin resistance with the positive effects on lipid metabolism through the action on HMG-CoA reductase and the striking effects on lipoprotein size and subclass particle concentrations [18].

IGF-1 decreased is related to reduced risk of all cancers, as already documented [19-21]. To date, studies suggest limiting protein consumption to achieve a reduction. From the results that emerge from our case, it would therefore not seem necessary to reduce or even eliminate proteins from dietary therapy [22], but it is necessary to use vegetable proteins.

The increase in SIRT1 is determined by weight loss $[23,24]$. SIRT1 is an antioxidant factor that causes further protection for T2DM and NAFLD [25].

Body composition changed with a marked reduction in VAT, which is directly correlated with the reduction of cardiovascular risk. Lean mass was preserved with subsequent preservation of muscle efficiency, thanks to the correct amount of protein $(1.4 \mathrm{~g} / \mathrm{kg})$.

The VLCKD was safe and no majorn side effects were reported. An increase in serum acid uric was observed (7,7 vs. $5.5 \mu \mathrm{mol} / \mathrm{L})$, despite therapy. As already found in the literature, this increase is due to the increase in soy content, present in meal replacements [26].

The gut microbiota composition is strictly influenced by the VLCKD and the source of dietary proteins modulates the variation of gut microbiota. In only 45 days we observed a reduction in Firmicutes, which are usually particularly abundant in obese subjects and are correlated with chronic low-grade inflammation, which supports the pathophysiological mechanisms underlying the mechanisms of insulin resistance. The most interesting aspect is the increase in Verrucomicrobia: Akkermansia muciniphila is the main genus of this phylum in human gut. An increase in Akkermansia $\mathrm{m}$. has been shown to lead to an improvement in barrier function, endotoxemia and 
insulin sensitivity, which could lead to protection from T2DM and all other MetS cofactors [27,28]. Further studies are needed to better clarify this increase and the role of this bacterium.

\section{Conclusions}

In conclusion, our report indicates that VLCKDs may hold promise as a strategy to improves parameters of metabolic syndrome, allowing a profound weight loss in patients with insulin resistance, obesity and MetS. This leads to a reduction in cardiovascular risk in only 45 days.

This is the first case-report showing a favourable effect of a VLCKD with veg proteins in gut microbiota.

The improvement of the Bacteroides/Firmicutes ratio and the increase in Verrucomicrobia could represent additional determining factors for choosing a VLCKD with vegan meal replacement in this type of patient.

Author Contributions: Author contributions to the paper were as follows. Study concept: S.B.; design: S.B.; acquisition of data: E.C., S.B., S.C., A.P.; interpretation of the data: S.M., C.L., R.R., M.C., L.G.; writing manuscript: E.C., S.B., A.P., L.G.; all authors approved the final version of the manuscript.

Funding: Financial support as well as the meal replacement protein preparations were kindly provided by New Penta s.r.l. (Cuneo, Italy). The funding source had no involvement in the study design, recruitment of patients, study interventions, data collection, or interpretation of the results.

Conflicts of Interest: The authors declare no conflict of interest.

\section{References}

1. Reaven, G.M. Banting lecture 1988. Role of insulin resistance in human disease. Diabetes 1988, 37, 15951607.

2. Chiarelli, F.; Mohn, A.; D'Adamo, E. La sindrome metabolica nel bambino. Il Diabete 2009, 21, 119-126.

3. Mottillo, S.; Filion, K.B.; Genest, J.; Joseph, L.; Pilote, L.; Poirier, P.; Rinfret, S.; Schiffrin, E.L.; Eisenberg, M.J. The metabolic syndrome and cardiovascular risk a systematic review and meta-analysis. J. Am. Coll. Cardiol. 2010, 56, 1113-1132, doi:10.1016/j.jacc.2010.05.034.

4. Gathirua-Mwangi, W.G.; Monahan, P.O.; Murage, M.J.; Zhang, J. Metabolic syndrome and total cancer mortality in the Third National Health and Nutrition Examination Survey. Cancer Causes Control 2017, 28, 127-136, doi:10.1007/s10552-016-0843-1.

5. Saklayen, M.G. The Global Epidemic of the Metabolic Syndrome. Curr. Hypertens. Rep. 2018, 20, 12, doi:10.1007/s11906-018-0812-z.

6. Caprio, M.; Infante, M.; Moriconi, E.; Armani, A.; Fabbri, A.; Mantovani, G.; Mariani, S.; Lubrano, C.; Poggiogalle, E.; Migliaccio, S.; et al. Very-low-calorie ketogenic diet (VLCKD) in the management of metabolic diseases: Systematic review and consensus statement from the Italian Society of Endocrinology (SIE). J. Endocrinol. Investig. 2019, 42, 1365-1386.

7. Feinman, R.D.; Pogozelski, W.K.; Astrup, A.; Bernstein, R.K.; Fine, E.J.; Westman, E.C.; Accurso, A.; Frassetto, L.; Gower, B.A.; McFarlane, S.I.; et al. Dietary carbohydrate restriction as the first approach in diabetes management: Critical review and evidence base. Nutrition 2015, 31, 1-13.

8. Goday, A.; Bellido, D.; Sajoux, I.; Crujeiras, A.B.; Burguera, B.; García-Luna, P.P.; Oleaga, A.; Moreno, B.; Casanueva, F.F. Short-term safety, tolerability and efficacy of a very low-calorie-ketogenic diet interventional weight loss program versus hypocaloric diet in patients with type 2 diabetes mellitus. Nutr. Diabetes 2016, 6, e230.

9. Dabke, K.; Hendrick, G.; Devkota, S. The gut microbiome and metabolic syndrome. J. Clin. Investig. 2019, 129, 4050-4057.

10. Turnbaugh, P.J.; Ley, R.E.; Mahowald, M.A.; Magrini, V.; Mardis, E.R.; Gordon, J.I. An obesity-associated gut microbiome with increased capacity for energy harvest. Nature 2006, 444, 1027-1031.

11. Xu, E.-T.; Nie, Y.-Z.; Yang, Z.; Lu, N.-H. The crosstalk between gut microbiota and obesity and related metabolic disorders. Future Microbiol. 2016, 11, 825-836.

12. Singh, R.K.; Chang, H.W.; Yan, D.; Lee, K.M.; Ucmak, D.; Wong, K.; Abrouk, M.; Farahnik, B.; Nakamura, M.; Zhu, T.H.; et al. Influence of diet on the gut microbiome and implications for human health. J. Transl. Med. 2017, 15, 73, doi:10.1186/s12967-017-1175-y. 
13. Festi, D.; Schiumerini, R.; Eusebi, L.H.; Marasco, G.; Taddia, M.; Colecchia, A. Gut microbiota and metabolic syndrome. World J. Gastroenterol. 2014, 20, 16079-16094, doi:10.3748/wjg.v20.i43.16079.

14. Paoli, A.; Canato, M.; Toniolo, L.; Bargossi, A.M.; Neri, M.; Mediati, M.; Alesso, D.; Sanna, G.; Grimaldi, K.A.; Fazzari, A.L.; et al. La dieta chetogenica: Un'opportunità terapeutica ignorata? Clin. Ter. 2011, 162, e137-146.

15. Basciani, S.; Costantini, D.; Contini, S. Safety and efficacy of a multiphase dietetic protocol with meal replacements including a step with very low calories diet. Endocrine 2015, 48, 863-870.

16. Basciani, S.; Camajani, E.; Contini, S.; Persichetti, A.; Risi, R.; Bertoldi, L.; Strigari, L.; Prossomariti, G.; Watanabe, M.; Mariani, S.; et al. Very-Low-Calorie Ketogenic Diets with Whey, Vegetable or Animal Protein in Patients with Obesity: A Randomized Pilot Study. J. Clin. Endocrinol. Metab. 2020, dgaa336, doi:10.1210/clinem/dgaa336.

17. Watanabe, M.; Tozzi, R.; Risi, R.; Tuccinardi, D.; Mariani, S.; Basciani, S.; Spera, G.; Lubrano, C.; Gnessi, L. Beneficial effects of the ketogenic diet on nonalcoholic fatty liver disease: A comprehensive review of the literature. Obes. Rev. 2020, 21, e13024, doi:10.1111/obr.13024.

18. Vergès, B. Pathophysiology of diabetic dyslipidaemia: Where are we? Diabetologia 2015, 58, 886-899.

19. Buono, R.; Longo, V.D. Starvation, Stress Resistance, and Cancer. Trends Endocrinol. Metab. 2018, 29, 271280, doi:10.1016/j.tem.2018.01.008.

20. Anisimov, V.N.; Bartke, A. The key role of growth hormone-insulin-IGF-1 signaling in aging and cancer. Crit. Rev. Oncol. Hematol. 2013, 87, 201-223, doi:10.1016/j.critrevonc.2013.01.005.

21. Wei, M.; Brandhorst, S.; Shelehchi, M.; Mirzaei, H.; Cheng, C.W.; Budniak, J.; Groshen, S.; Mack, W.J.; Guen, E.; Di Biase, S.; et al. Fasting-mimicking diet and markers/risk factors for aging, diabetes, cancer, and cardiovascular disease. Sci. Transl. Med. 2017, 9, eaai8700, doi:10.1126/scitranslmed.aai8700.

22. Levine, M.E.; Suarez, J.A.; Brandhorst, S.; Balasubramanian, P.; Cheng, C.W.; Madia, F.; Fontana, L.; Mirisola, M.G.; Guevara-Aguirre, J.; Wan, J.; et al. Low protein intake is associated with a major reduction in IGF-1, cancer, and overall mortality in the 65 and younger but not older population. Cell Metab. 2014, 19, 407-417, doi:10.1016/j.cmet.2014.02.006.

23. Mariani, S.; di Giorgio, M.R.; Martini, P.; Persichetti, A.; Barbaro, G.; Basciani, S.; Contini, S.; Poggiogalle, E.; Sarnicola, A.; Genco, A.; et al. Inverse Association of Circulating SIRT1 and Adiposity: A Study on Underweight, Normal Weight, and Obese Patients. Front. Endocrinol. (Lausanne) 2018, 9, 449, doi:10.3389/fendo.2018.00449. PMID: 30131769; PMCID: PMC6090043.

24. Mariani, S.; Costantini, D.; Lubrano, C.; Basciani, S.; Caldaroni, C.; Barbaro, G.; Poggiogalle, E.; Donini, L.M.; Lenzi, A.; Gnessi, L. Circulating SIRT1 inversely correlates with epicardial fat thickness in patients with obesity. Nutr. Metab. Cardiovasc. Dis. 2016, 26, 1033-1038, doi:10.1016/j.numecd.2016.06.001.

25. Mariani, S.; Fiore, D.; Basciani, S.; Persichetti, A.; Contini, S.; Lubrano, C.; Salvatori, L.; Lenzi, A.; Gnessi, L. Plasma levels of SIRT1 associate with non-alcoholic fatty liver disease in obese patients. Endocrine 2015, 49, 711-716, doi:10.1007/s12020-014- 0465-x.

26. Schmidt, J.A.; Crowe, F.L.; Appleby, P.N.; Key, T.J.; Travis, R.C. Serum uric acid concentrations in meat eaters, fish eaters, vegetarians and vegans: A cross-sectional analysis in the EPIC-Oxford cohort. PLoS ONE 2013, 8, e56339, doi:10.1371/journal.pone.0056339.

27. Fan, Y.; Pedersen, O. Gut microbiota in human metabolic health and disease. Nat. Rev. Microbiol. 2020, doi:10.1038/s41579-020-0433-9.

28. Depommier, C.; Everard, A.; Druart, C.; Plovier, H.; Van Hul, M.; Vieira-Silva, S.; Falony, G.; Raes, J.; Maiter, D.; Delzenne, N.M.; et al. Supplementation with Akkermansia muciniphila in overweight and obese human volunteers: A proof-of-concept exploratory study. Nat. Med. 2019, 25, 1096-1103, doi:10.1038/s41591-019-0495-2.

Publisher's Note: MDPI stays neutral with regard to jurisdictional claims in published maps and institutional affiliations.

(C) 2020 by the authors. Submitted for possible open access publication under the terms and conditions of the Creative Commons Attribution (CC BY) license (http://creativecommons.org/licenses/by/4.0/). 